\title{
Cassini observations of the thermal plasma in the vicinity of Saturn's main rings and the $F$ and $G$ rings
}

\author{
R. L. Tokar, ${ }^{1}$ R. E. Johnson, ${ }^{2}$ M. F. Thomsen, ${ }^{1}$ D. M. Delapp, ${ }^{1}$ R. A. Baragiola, ${ }^{2}$ \\ M. F. Francis, ${ }^{2}$ D. B. Reisenfeld, ${ }^{3}$ B. A. Fish, ${ }^{3}$ D. T. Young, ${ }^{4}$ F. J. Crary ${ }^{4}$ A. J. Coates, ${ }^{5}$ \\ D. A. Gurnett, ${ }^{6}$ and W. S. Kurth ${ }^{6}$
}

Received 11 February 2005; revised 3 May 2005; accepted 16 May 2005; published 11 June 2005.

[1] The ion mass spectrometer on Cassini detected enhanced ion flux near Saturn's main rings that is consistent with the presence of atomic and molecular oxygen ions in the thermal plasma. The ring "atmosphere" and "ionosphere" are likely produced by UV photosputtering of the icy rings and subsequent photoionization of $\mathrm{O}_{2}$. The identification of the $\mathrm{O}^{+}$and $\mathrm{O}_{2}^{+}$ions is made using time-of-flight analysis and densities and temperatures are derived from the ion counting data. The ion temperatures over the main rings are a minimum near synchronous orbit and increase with radial distance from Saturn as expected from ion pick up in Saturn's magnetic field. The $\mathrm{O}_{2}^{+}$temperatures provide an estimate of the neutral $\mathrm{O}_{2}$ temperature over the main rings. The ion mass spectrometer also detected significant $\mathrm{O}_{2}^{+}$outside of the main rings, near the $\mathrm{F}$ ring. It is concluded that between the $F$ and $G$ rings, the heavy ion population most likely consists of an admixture of $\mathrm{O}_{2}^{+}$and water group ions $\mathrm{O}^{+}$, $\mathrm{OH}^{+}$, and $\mathrm{H}_{2} \mathrm{O}^{+}$. Citation: Tokar, R. L., et al. (2005), Cassini observations of the thermal plasma in the vicinity of Saturn's main rings and the F and G rings, Geophys. Res. Lett., 32, L14S04, doi:10.1029/2005GL022690.

\section{Introduction}

[2] During orbit insertion about Saturn on July 1, 2004, the Cassini spacecraft passed over the $\mathrm{B}, \mathrm{A}$, and $\mathrm{F}$ rings and the Cassini division before descending, inside the $\mathrm{G}$ ring, through the ring plane. The trajectory provided the first in-situ plasma measurements over Saturn's main rings, complementing data obtained by the Voyager 2 plasma science (PLS) instrument outside the main rings at a minimum radial distance of about $2.85 \mathrm{R}_{\mathrm{s}}\left(1 \mathrm{R}_{\mathrm{s}}=\right.$ 1 Saturn radii $=60,330 \mathrm{~km}$ ). See Richardson [1998] for a review of the Voyager PLS data. The ion mass spectrometer (IMS), a component of the Cassini plasma spectrometer (CAPS) [Young et al., 2004], observed ion flux over the

\footnotetext{
${ }^{1}$ Space Science and Applications, Los Alamos National Laboratory, Los Alamos, New Mexico, USA.

${ }^{2}$ Department of Materials Science and Engineering, University of Virginia, Charlottesville, Virginia, USA.

${ }^{3}$ Department of Physics and Astronomy, University of Montana, Missoula, Montana, USA.

${ }^{4}$ Southwest Research Institute, San Antonio, Texas, USA

${ }^{5}$ Mullard Space Science Laboratory, University College London, Surrey, UK.

${ }^{6}$ Department of Physics and Astronomy, University of Iowa, Iowa City, Iowa, USA.
}

Copyright 2005 by the American Geophysical Union. 0094-8276/05/2005GL022690 main rings consistent with the presence of atomic and molecular oxygen ions [Young et al., 2005]. In addition, the ion neutral mass spectrometer (INMS) on Cassini also found evidence for $\mathrm{O}^{+}, \mathrm{O}_{2}^{+}$, and $\mathrm{H}^{+}$, although these data were obtained a few minutes after the CAPS observations [Waite et al., 2005]. An $\mathrm{O}_{2}$ ring atmosphere was suggested to be present due to UV photodissociation of water vapor produced by meteoroid impact [Ip, 1995], but it is more likely produced by UV photosputtering of the icy ring particles [Westley et al., 1995; R. E. Johnson et al., Production, ionization, and redistribution of Saturn's $\mathrm{O}_{2}$ ring atmosphere, submitted to Icarus, 2005, hereinafter referred to as Johnson et al., submitted manuscript, 2005]. The ionosphere observed here is produced by photoionization of $\mathrm{O}_{2}$. Complementary electron observations by CAPS near the rings are described by Coates et al. [2005].

[3] In this study, the IMS data over the main rings are further analyzed, extending the results by Young et al. [2005]. The identification of the $\mathrm{O}^{+}$and $\mathrm{O}_{2}^{+}$ion species over the main rings is presented using IMS time-of-flight (TOF) analysis, and densities and temperatures of the oxygen species are derived. In addition, IMS observed enhanced ion flux as Cassini crossed the ring plane, outside of the main rings and in the vicinity of the $F$ and $G$ rings. Near the edge of the main rings, the IMS ion flux as a function of energy per charge provides strong evidence for the presence of $\mathrm{O}_{2}^{+}$. It is concluded that the enhanced ion flux between the $\mathrm{F}$ and $\mathrm{G}$ rings consists of water group ions $\mathrm{O}^{+}, \mathrm{OH}^{+}$, and $\mathrm{H}_{2} \mathrm{O}^{+}$admixed with $\mathrm{O}_{2}^{+}$.

\section{CAPS IMS Data Analysis}

[4] Enhanced ion flux is observed by IMS in two distinct time intervals on July 1, 2004, both occurring when individual IMS detectors are looking into the direction of corotating plasma flow. The geometry of the Cassini trajectory near the rings in a plane parallel and perpendicular to the Saturn spin axis is shown schematically in Figure 1. Also shown are the approximate positions of the B, A, F, and $G$ rings along with the Cassini division. The two time intervals of enhanced ion flux measured by IMS are denoted in red on the trajectory. Note that in the first time interval, Cassini ranges from about 0.15 to $0.25 \mathrm{R}_{\mathrm{s}}$ north of the main rings, while the second interval of enhanced ion counts contains the ring plane crossing inside the $\mathrm{G}$ ring. Outside of the main rings and throughout the second time interval, Cassini is in the region of trapped radiation belt particles (entered at time $t_{3}$ ) and these particles penetrate IMS and produce a large nearly isotropic background signal. For 


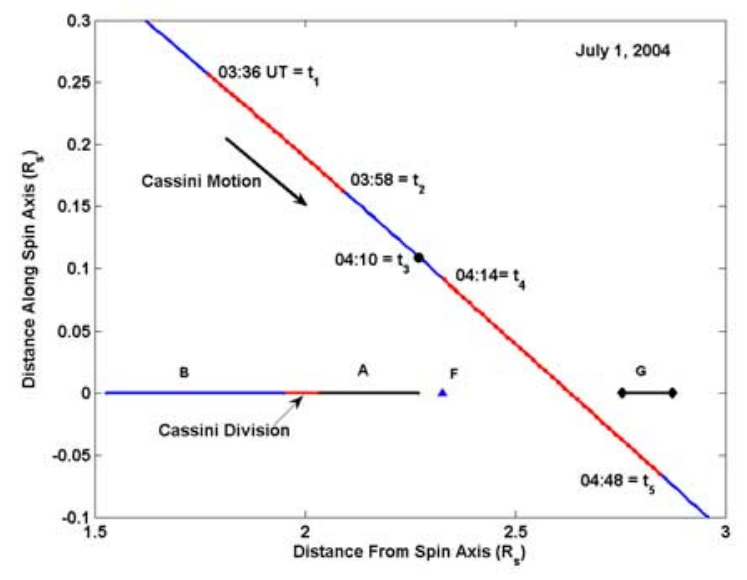

Figure 1. Schematic of the Cassini trajectory near the rings. Enhanced ion flux is observed in the regions colored red when IMS was viewing into the corotating plasma flow. Cassini entered the penetrating radiation belts at time $t_{3}$.

reference, the Cassini INMS measurements of $\mathrm{H}^{+}, \mathrm{O}^{+}$, and $\mathrm{O}_{2}^{+}$were obtained from $\sim 04: 00$ UT to $\sim 04: 06: 30$ UT, between $t_{2}$ and $t_{3}$.

[5] In order to extract plasma parameters from the IMS data, it is necessary to establish the ion composition of the plasma. The first time interval over the main rings will be the focus in this study. Indirect evidence exists for the presence of $\mathrm{O}^{+}$and $\mathrm{O}_{2}^{+}$between the times $\mathrm{t}_{1}$ and $\mathrm{t}_{2}$. A model thermal plasma corotating with Saturn and containing ions with mass per charge of $16\left(\right.$ e.g. $\mathrm{O}^{+}$) and 32 (e.g. $\mathrm{O}_{2}^{+}$) reproduces many features in the observed ion flux. For example, the elevated ion flux is only observed when the IMS detectors view into the corotation direction and the observed ion energies in the IMS frame are consistent with corotating ions having mass per charge of 16 and 32. Note that these data are not conclusive enough to exclude $\mathrm{OH}^{+}$or $\mathrm{H}_{2} \mathrm{O}^{+}$, because the mass per charge of these species is close to that of $\mathrm{O}^{+}$. In addition to this indirect evidence, the presence of $\mathrm{O}^{+}$and $\mathrm{O}_{2}^{+}$is indicated by analysis of IMS time-of-flight (TOF) spectra, shown in Figure 2. The data (black points) are measured between the times $t_{1}$ and $t_{2}$ at the energies 14.7 and $29.5 \mathrm{eV}$ and are compared to model spectra (red lines). The model spectra are derived from ion-optics simulations of $\mathrm{O}^{+}$and $\mathrm{O}_{2}^{+}$trajectories through the IMS instrument [e.g., see Young et al., 2004]. The relatively good agreement between the measured and simulated spectra enables the identification of $\mathrm{O}^{+}$and $\mathrm{O}_{2}^{+}$ over the rings. From the simulations, it is established that $\mathrm{O}^{+}$ions contribute nearly equal to the peaks in the TOF at $600 \mathrm{~ns}$ (peak 1) and $800 \mathrm{~ns}$ (peak 2), whereas $\mathrm{O}_{2}^{+}$contributes essentially only to peak 2 .

[6] The TOF data shown in Figure 2 at $14.7 \mathrm{eV}$ is consistent with $\sim 100 \% \mathrm{O}^{+}$while the $29.5 \mathrm{eV}$ spectrum is consistent with an admixture of $\mathrm{O}^{+}(\sim 18 \%)$ and $\mathrm{O}_{2}^{+}$ ( $\sim 82 \%)$. Higher energy spectra at $42 \mathrm{eV}$ (not shown) are consistent with $100 \% \mathrm{O}_{2}^{+}$. These results are in agreement with the ion mass per charge counting data and the predictions of a corotating thermal plasma model. In addition, it should be noted that the IMS TOF data is not consistent with significant amounts of $\mathrm{H}^{+}$or $\mathrm{H}_{2}^{+}$, and also does not rule out admixtures of $\mathrm{OH}^{+}$or $\mathrm{H}_{2} \mathrm{O}^{+}$. For this reason, it is important to note that this study uniquely identifies $\mathrm{O}_{2}^{+}$over the main rings but the TOF data identifying $\mathrm{O}^{+}$could also be modeled as a mixture of $\mathrm{OH}^{+}$and/or $\mathrm{H}_{2} \mathrm{O}^{+}$. Additional peaks in the TOF spectra arise from secondary electrons ejected from various electrodes by particles scattered in the IMS foils, such as the peaks at $\sim 100$ ns and $\sim 300-400$ ns. These 'ghost' peaks are visible in Figure 2 but not discussed in detail here.

[7] To extract densities and temperatures for $\mathrm{O}^{+}$and $\mathrm{O}_{2}^{+}$ over the main rings, it is assumed here that the plasma phase space density can be modeled as the sum of two isotropic convected maxwellian distributions. The look direction of IMS was predominantly perpendicular to the magnetic field direction for all of the ion data obtained over the main rings; therefore, measurements of the full ion phase space density are not available. This precludes the investigation of ion temperature anisotropies, as studied by Gan-Baruch et al. [1994]. Consistent with the discussion above, the convection velocity is taken as the rigid plasma corotation velocity in the moving IMS frame, leaving two free parameters for each phase space distribution, species density and temperature. For each of the eight IMS detectors and their unique look directions, a forward modeling procedure is used to calculate ion counting rates along the Cassini trajectory as a function of energy per charge and the two species densities and temperatures. The free parameters $\left(\mathrm{N}_{\mathrm{O}+}, \mathrm{T}_{\mathrm{O}+}, \mathrm{N}_{\mathrm{O} 2+}, \mathrm{T}_{\mathrm{O} 2+}\right)$ are determined by minimizing the RMS difference in the calculated and measured ion counts as a function of energy per charge. Figure 3 illustrates a sample result of this procedure. The IMS data is measured at $\sim 03: 46 \mathrm{UT}$, when Cassini was $\sim 0.22 \mathrm{R}_{\mathrm{S}}$ above the magnetic equator and at an equatorial radius of $\sim 1.91 \mathrm{R}_{\mathrm{S}}$. The data are the black points at the energy per charge values of the IMS electrostatic analyzer. The forward modeling procedure results in the $\mathrm{O}^{+}$and $\mathrm{O}_{2}^{+}$count distributions (blue and green, respectively) that sum to match the measured counts. This is a

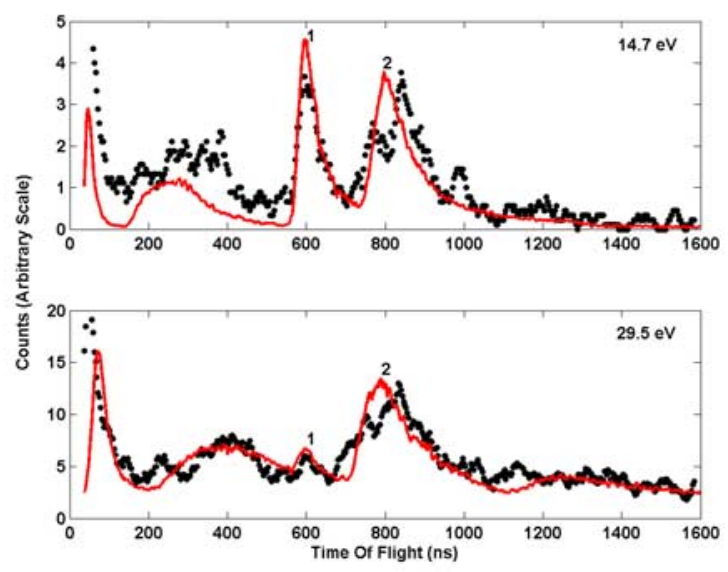

Figure 2. IMS time of flight data for two energies summed over the time interval $t_{1}$ to $t_{2}$ in Figure 1 . The model calculations shown in red establish the presence of $\mathrm{O}^{+}$and $\mathrm{O}_{2}^{+}$over the main rings although the additional presence of $\mathrm{OH}^{+}$and $\mathrm{H}_{2} \mathrm{O}^{+}$cannot be excluded. 


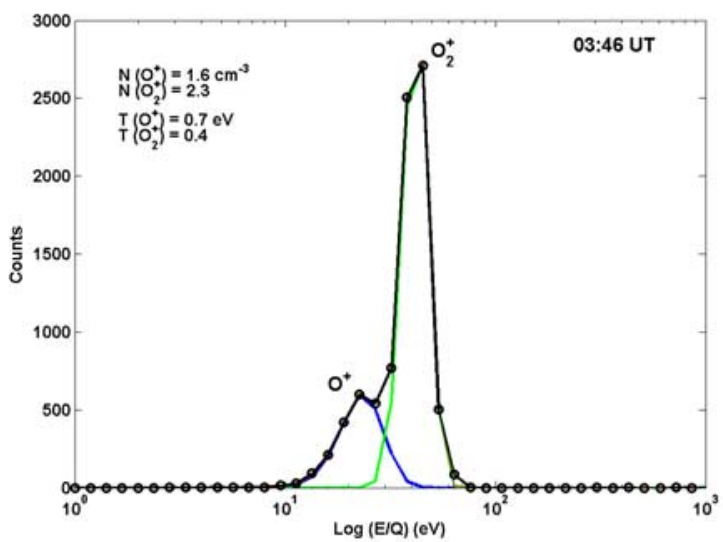

Figure 3. Sample fits to both $\mathrm{O}^{+}$and $\mathrm{O}_{2}^{+}$ion counting data over the main rings using the corotating forward model.

typical result between times $t_{1}$ and $t_{2}$ over the main rings and illustrates the ability of the model to match the observations.

[8] Figure 4 illustrates the results of this procedure for the time interval $t_{1}$ to $t_{2}$. Shown are the densities of $\mathrm{O}^{+}$and $\mathrm{O}_{2}^{+}$ plotted as a function of radial distance along the magnetic equator. In addition, the total electron density obtained from an analysis of plasma wave emissions observed by the Cassini RPWS [Gurnett et al., 2005] is also plotted. A striking feature in these data is the rapid increase in both the ion and electron densities that begins near $1.85 \mathrm{R}_{\mathrm{S}}$. This is approximately the radial distance from Saturn where the orbital speed in Saturn's gravitational field is equal to the speed of rigid corotation with Saturn (synchronous orbit, $\sim 1.85 \mathrm{R}_{\mathrm{S}}$ ). While still over the B-ring, the densities increase as the spacecraft distance above the ring plane slowly decreases (see Figure 1) until both the $\mathrm{O}^{+}$and $\mathrm{O}_{2}^{+}$densities are $\sim 2 \mathrm{~cm}^{-3}$ with the total ion density $\sim 4 \mathrm{~cm}-{ }^{3}$. Note that the IMS derived peak ion density is about a factor of

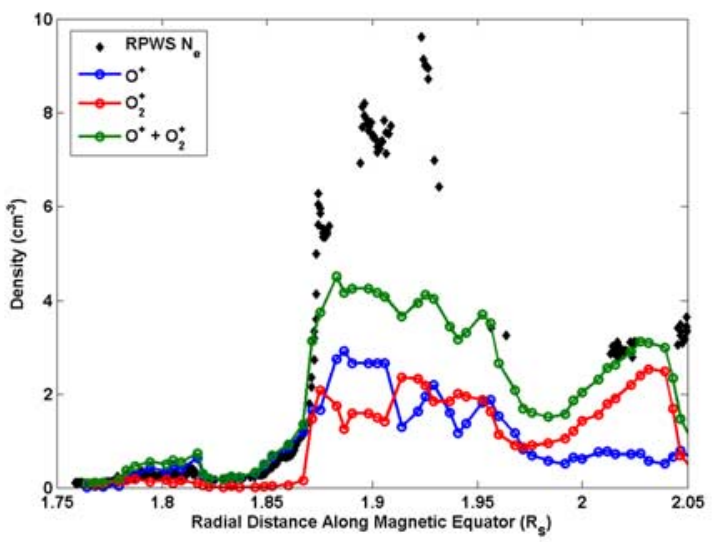

Figure 4. Densities of $\mathrm{O}^{+}$and $\mathrm{O}_{2}^{+}$, together with the RPWS electron density, over the main rings.
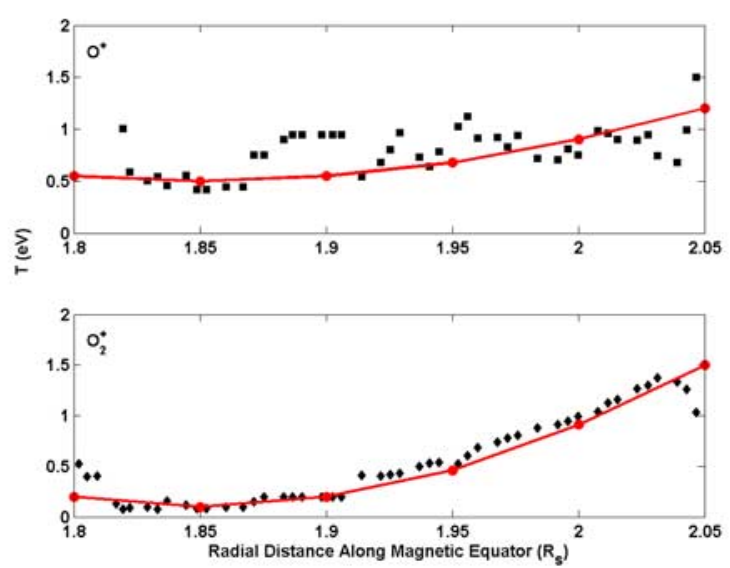

Figure 5. Temperature data (black points) for $\mathrm{O}^{+}$and $\mathrm{O}_{2}^{+}$ over the main rings, together with the predicted temperatures for ion pick-up in Saturn's corotating magnetic field (red curves).

2 lower than the RPWS peak electron densities but in contrast the ion and electron densities are in fairly good agreement for lower values $\left(<\sim 4 \mathrm{~cm}^{-3}\right)$. The electrostatic potential of Cassini is estimated to be negative $(\sim-1.5 \mathrm{eV})$ at these times, therefore any lighter ions e.g. $\mathrm{H}^{+}$or $\mathrm{H}_{2}^{+}$ should be detected by the IMS. However, as discussed previously, there is no evidence for light ions over the main rings in the IMS data, although there is in the Cassini INMS data at somewhat later observation times. This discrepancy between the IMS and RPWS results is under investigation.

[9] The analysis of the ion data over the main rings also provides estimates for the $\mathrm{O}^{+}$and $\mathrm{O}_{2}^{+}$temperatures. These are shown in Figure 5, with the top panel displaying the $\mathrm{O}^{+}$ temperature and the bottom $\mathrm{O}_{2}^{+}$. Both the $\mathrm{O}^{+}$and $\mathrm{O}_{2}^{+}$ temperatures are a minimum near synchronous orbit, $\sim 1.85 \mathrm{R}_{\mathrm{S}}$, and increase with radial distance beyond synchronous orbit. This is suggestive of an ion pick up process in the corotating magnetic field of Saturn, further supported by model calculations (red curves) for the expected temperatures of ions picked up in the corotating magnetic field. The calculated temperature has two contributions, the initial temperature of the ion when formed, and an effective temperature obtained from the gyrational kinetic energy of the orbiting ion when it is picked up by the corotating magnetic field. Over the rings, $\mathrm{O}_{2}$ molecules are photoionized to produce $\mathrm{O}_{2}^{+}$and $\mathrm{O}^{+}$in a ratio of about 3 or 4 to 1 depending on solar activity. The $\mathrm{O}_{2}^{+}$is formed relatively cold but the $\mathrm{O}^{+}$, due to the ionization plus dissociation process, is formed exothermically. The comparison of this simple model with the IMS data suggest that the $\mathrm{O}^{+}$is formed with a temperature $\sim 0.5 \mathrm{eV}$, and that the $\mathrm{O}_{2}^{+}$is formed relatively cold, with temperature $\sim 0.10 \mathrm{eV}$. The $\mathrm{O}^{+}$formation temperature is consistent with experiments on ionization and dissociation, while the $\mathrm{O}_{2}^{+}$formation temperature obtained from the IMS data $(\sim 0.1 \mathrm{eV})$ provides an estimate of the temperature of the neutral atmosphere above the rings. The extracted temperature is higher than that expected $(\sim 2 \mathrm{kT})$ when the $\mathrm{O}_{2}$ repeatedly 


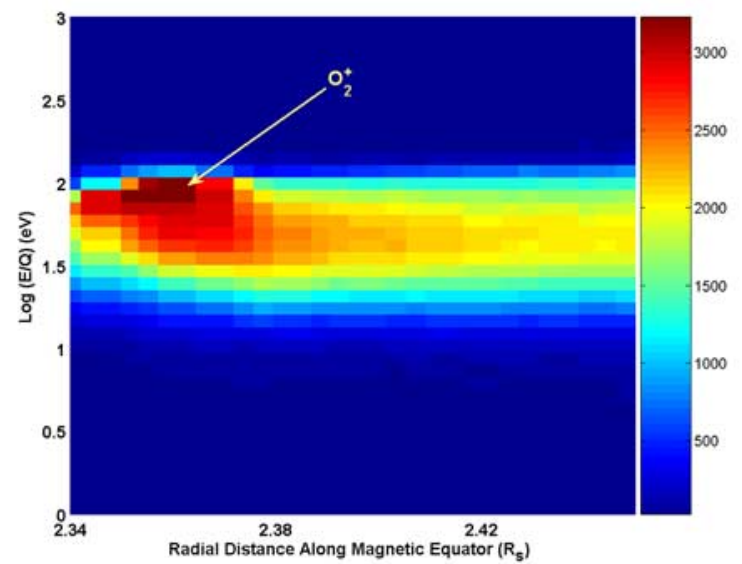

Figure 6. IMS counting data as a function of energy per charge and position measured as Cassini moves outward from the edge of the main rings. There is a clear signature of $\mathrm{O}_{2}^{+}$in the vicinity of the $\mathrm{F}$ ring.

adsorb on and desorb from the ring particles which have temperatures, $\mathrm{T} \sim 80$ to $100 \mathrm{~K}$. Although the extracted $\mathrm{O}_{2}$ temperature has a large uncertainty, this may be suggestive of more energetic surfaces processes or to the fact that ionization occurs primarily off the magnetic equator (Johnson et al., submitted manuscript, 2005).

[10] The second time interval of enhanced ion flux in IMS is between the times $t_{4}$ and $t_{5}$ in Figure 1, measured as Cassini was outside the main rings and descending through the ring plane between the $\mathrm{F}$ and G-rings. Because Cassini entered the environment of fast radiation belt particles at $t_{3}$, a large background signal was present in the IMS TOF data making identification via the IMS TOF data difficult. However the IMS ion data does indicate the presence of $\mathrm{O}_{2}^{+}$, in addition to the expected water group ions (see below), at early times in this interval. This is shown in Figure 6, where the IMS ion counting data for detector number 8 near the time $t_{4}$ are plotted as a function of energy per charge and radial distance along the magnetic equator. The penetrating background is removed from this data by subtracting the average observed counts from about 10 to $20 \mathrm{keV}$. Similar to IMS data over the main rings [Young et al., 2005], enhanced counts at the expected $\mathrm{O}_{2}^{+}$energies are visible in Figure 6. Note that Cassini was changing orientation just prior to the peak in ion counts denoted in the figure, bringing IMS detector 8 viewing into the direction of corotational flow. This is the most distinct $\mathrm{O}_{2}^{+}$signature observed during the time $\mathrm{t}_{4}$ to $\mathrm{t}_{5}$, although $\mathrm{O}_{2}^{+}$could exist at lower relative densities throughout the interval. The full analysis of these data is beyond the scope of this study. Briefly, the initial analysis has focused on two models, the first a two species model of lighter and heavier ions (e.g. $\mathrm{O}^{+}$ and $\mathrm{O}_{2}^{+}$) like that used over the main rings. Fits of the IMS flux using this model are relatively good and result in the two species having about equal densities. The second model assumes water group ions, e.g. $\mathrm{O}^{+}, \mathrm{OH}^{+}$, and $\mathrm{H}_{2} \mathrm{O}^{+}$, together with the addition of a minor species heavier ion, e.g. $\mathrm{O}_{2}^{+}$. The water group ions are assumed to have the relative concentrations given by Richardson and Jurac [2004]. This model is also similar to model concentrations obtained by $\mathrm{Ip}$ [2000] including $\mathrm{O}_{2}^{+}$. For the region outside of the peak seen in Figure 6, the heavy ion is always a minor species and relatively good fits to the IMS flux throughout the region are found. Although further analysis is required on these data, the preliminary conclusion is that the second model appears more likely due to the absence of a prominent heavy ion signature in the IMS ion flux for most of the region between the F and G-rings. It should also be noted that throughout this region, the Cassini electrostatic potential is estimated from the CAPS electron spectrometer to be positive, in the range of a few eV. This may account for the lack of significant corotating light ions, e.g. $\mathrm{H}^{+}$or $\mathrm{H}_{2}^{+}$, evident in the IMS ion flux as these ions would be repelled by IMS.

\section{Discussion}

[11] The analysis of the IMS data presented here confirms the detection of atomic and molecular oxygen ions over Saturn's main rings, with particular emphasis placed on the positive identification of $\mathrm{O}_{2}^{+}$over the main rings obtained from the IMS TOF data. In addition, we report the first detection of molecular oxygen ions in thermal plasma just outside the main rings, in the vicinity of the F-ring. As the spacecraft moved radially outward toward the G-ring, the thermal plasma is assumed to be roughly described as a plasma formed from water vapor with, likely, a component of $\mathrm{O}_{2}^{+}$. Ion temperatures are extracted from the IMS data over the main rings and indicate ion pick up in Saturn's corotating magnetic field is the dominant energy source away from synchronous orbit. The ion composition over the main rings is suggestive of the presence of a Europa-like molecular oxygen atmosphere produced by the decomposition of water [Young et al., 2005; Johnson et al., submitted manuscript, 2005]. However, unlike at Europa, the $\mathrm{O}_{2}$ must be produced by UV photons and not by the energetic plasma, which has a very small flux over the main rings. In the region outside of the main rings the energetic particle flux is large. Therefore, the source of the thermal plasma can be sputtering and decomposition of ice grains associated with the $\mathrm{F}$ and G-rings by the hot plasma. Additional sources in this region are the ejection of material from the main rings and diffusion of plasma inward from the region where Enceladus, Mimas and the E-ring are important sources of plasma. The detection of $\mathrm{O}_{2}^{+}$close to the edge of the main rings is suggestive of a contribution from a ring source, in addition to the local sources (Johnson et al., submitted manuscript, 2005). Detailed modeling will be required to confirm these possibilities.

[12] Acknowledgments. We wish to thank the large number of scientists and engineers on the CAPS team who made the results reported here possible. The work at Los Alamos was performed under the auspices of the US DOE and was supported by the NASA Cassini program. The ion mass spectrometer is a component of the Cassini Plasma Spectrometer and was supported by JPL contract 1243218 with Southwest Research Institute. Cassini is managed by the Jet Propulsion Laboratory for NASA.

\section{References}

Coates, A. J., et al. (2005), Plasma electrons above Saturn's main rings: CAPS observations, Geophys. Res. Lett., doi:10.1029/2005GL022694, in press.

Gan-Baruch, Z., A. Eviatar, J. D. Richardson, and R. L. McNutt Jr. (1994), Plasma observations in the ring plane of Saturn, J. Geophys. Res., 99, $11,063-11,077$. 
Gurnett, D. A., et al. (2005), Radio and plasma wave observations at Saturn from Cassini's approach and first orbit, Science, 307, 1255-1259, doi:10.1126/science.1105356.

Ip, W. H. (1995), The exospheric systems of Saturn's rings, Icarus, 115, $295-303$

Ip, W. H. (2000), Thermal plasma composition in Saturn's magnetosphere, Planet. Space Sci., 48, 775-783

Richardson, J. D. (1998), Thermal plasma and neutral gas in Saturn's magnetosphere, Rev Geophys., 36, 501-524.

Richardson, J. D., and S. Jurac (2004), A self-consistent model of plasma and neutrals at Saturn: The ion tori, Geophys. Res. Lett., 31, L24803, doi:10.1029/2004GL020959.

Waite, J. H., Jr., et al. (2005), Cassini ion and neutral mass spectrometer measurements of oxygen ions near Saturn's A-Ring, Science, 307, 1260 1262, doi:10.1126/science.1105734.

Westley, M. S., R. A. Baragiola, R. E. Johnson, and G. A. Baratta (1995), Ultraviolet photodesorption from water ice, Planet. Space Sci., 43, $1311-1315$.

Young, D. T., et al. (2004), Cassini plasma spectrometer investigation, Space Sci. Rev., 114, 1-112.
Young, D. T., et al. (2005), Composition and dynamics of plasma in Saturn's magnetosphere, Science, 307, 1262-1266, doi:10.1126/ science. 1106151.

R. A. Baragiola, M. F. Francis, and R. E. Johnson, Department of Materials Science and Engineering, University of Virginia, Thornton Hall B103, Charlottesville, VA 22903, USA.

B. A. Fish and D. B. Reisenfeld, Department of Physics and Astronomy, University of Montana, Missoula, MT 59812, USA.

A. J. Coates, Mullard Space Science Laboratory, University College London, Surrey RH5 6NT, UK.

F. J. Crary and D. T. Young, Southwest Research Institute, San Antonio, TX 78228-0510, USA.

D. M. Delapp, M. F. Thomsen, and R. L. Tokar, Space Science and Applications, Los Alamos National Laboratory, Los Alamos, NM 87545, USA. (rlt@lanl.gov)

D. A. Gurnett and W. S. Kurth, Department of Physics and Astronomy, University of Iowa, Iowa City, IA 52242, USA 